\title{
Label-free and real-time detection of specific binding of IgG proteins by oblique-incidence reflectivity difference method
}

\author{
SUN Yue ${ }^{1,2}$, HE LiPing ${ }^{1,2}$, DAI Jun ${ }^{2}$, WANG JingYi ${ }^{2}$, LÜ HuiBin ${ }^{2 *}$, WANG ShuFang ${ }^{1 *}$, \\ JIN KuiJuan ${ }^{2}$, ZHOU YueLiang ${ }^{2} \&$ YANG GuoZhen ${ }^{2}$ \\ ${ }^{1}$ College of Physics Science and Technology, Hebei University, Baoding 071002, China; \\ ${ }^{2}$ Beijing National Laboratory for Condensed Matter Physics, Institute of Physics, Chinese Academy of Sciences, Beijing 100190, China
}

Received February 13, 2012; accepted March 26, 2012

\begin{abstract}
The entire interaction process between anti-mouse immunoglobulin-G (IgG) and mouse $\operatorname{IgG}$ with a concentration range from 1 to $0.25 \mathrm{mg} / \mathrm{mL}$ has been label-free detected by the oblique incidence reflectivity difference (OIRD) method. We achieved the two-dimensional scanning images of OIRD $\operatorname{Re}\left\{\Delta_{\mathrm{p}}-\Delta_{\mathrm{s}}\right\}$ intensities before and after interactions as well as the curves of reaction dynamic processes. The experimental results suggest that OIRD method not only can label-free distinguish the different concentrations of proteins and detect biological microarrays, but also can real-time monitor the kinetic processes of biomolecular interactions.
\end{abstract}

oblique-incidence reflectivity difference (OIRD), label-free and real-time detection, biomolecular interactions

Citation: Sun Y, He L P, Dai J, et al. Label-free and real-time detection of specific binding of IgG proteins by oblique-incidence reflectivity difference method. Chin Sci Bull, 2012, 57: 2898-2900, doi: 10.1007/s11434-012-5263-3

It is still a big challenge to obtain a label-free and high throughput detection method for biological microarrays, because up to now, the label-based detection methods, such as radioactive labeling, chemiluminescence, and fluorescence, are widely applied in the monitoring of biomolecular interactions for their high sensitivity and high throughput $[1,2]$. However, the whole procedures including labeling and detecting are very costly and time-consuming. Especially, the extrinsic label probably impacts the biomolecular characteristics and the natural activities $[3,4]$. So far, though the surface plasmon resonance (SPR) is the most widely used method that has been applied successfully to the detection of label-free biological microarrays [5,6], SPR has not been considered to be a high-throughput method yet. In addition, label-free and real-time detection of dynamic processes of biomolecular interactions can obtain the quantitative information of interaction kinetics; therefore, label-free and real-time detection is more attractive for biological

*Corresponding authors (email: hblu@iphy.ac.cn; sfwang_researcher@yahoo.com.cn) investigation. Oblique-incidence reflectivity difference (OIRD) method as a high sensitive and label-free technique has been used in the detection of biological microarrays [7]. We have reported the label-free detection of biomolecular microarrays and real-time detection of biomolecular interactions using OIRD method [8-14]. In this work, we report on the label-free and real-time detection of interaction processes of IgG proteins by OIRD method. The experimental results suggest that the OIRD method can measure the entire interaction process of biological molecules and has potential application for the label-free detection of biological microarrays.

\section{Experimental}

Figure 1 shows the schematic diagram of OIRD setup for the label-free and real-time detection of $\mathrm{IgG}$ proteins. As mentioned in our previous work [11], the probe beam is a p-polarized He-Ne laser with $632.8 \mathrm{~nm}$ wavelength, and 


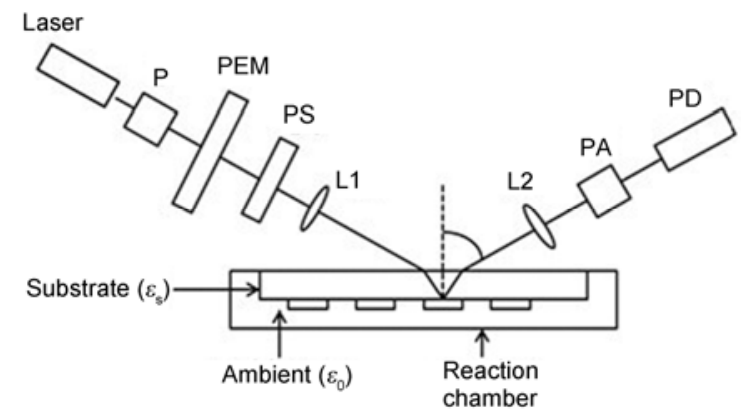

Figure 1 The schematic diagram of OIRD setup for the label-free and real-time detection of IgG protein microarray. Laser, a $7 \mathrm{~mW}$ He-Ne laser; P, polarizer; PEM, photoelastic modulator; PS, 1/2 plate; L1 and L2, lens; $\mathrm{PA}$, polarization analyzer; $\mathrm{PD}$, photodiode detector.

first passes through a photoelastic modulator (PEM90) which induces the probe beam to oscillate between $\mathrm{p}$ - and s-polarization with the modulated frequency $\Omega=50 \mathrm{kHz}$. Then the modulated polarized light passes through a phase shifter (PS), which causes a variable phase between $\mathrm{p}$ - and s-polarization components, and is focused on the surface of protein microarray at an incident angle of $60^{\circ}$. The protein microarray is covered on the chamber and the sample surface is on contact with the liquid phase. The incident light is reflected by the sample surface, and the intensity of resultant beam is detected by a silicon photodiode (PD) and two lock-in amplifiers. At oblique incidence, for the different surface, the surface reflectivities of p- and s-polarized light are different as the different of the surface layer thickness and/or the complex dielectric constant. Let us define $r_{\mathrm{p} 0}$ and $r_{\mathrm{s} 0}$ as the reflectivities from the bare microarray surface without protein molecules for $\mathrm{p}$ - and s-polarized light, and $r_{\mathrm{p}}$ and $r_{\mathrm{s}}$ be the reflectivities from the protein covered surface, respectively. The changes of $\mathrm{p}$ - and s-components in reflectivity are defined as $\Delta_{\mathrm{p}}=\left|\left(r_{\mathrm{p}}-r_{\mathrm{p} 0}\right) / r_{\mathrm{p} 0}\right|$ and $\Delta_{\mathrm{s}}=\mid\left(r_{\mathrm{s}}-\right.$ $\left.r_{\mathrm{s} 0}\right) / r_{\mathrm{s} 0}$, respectively. The difference in reflectivity change is $\Delta_{\mathrm{p}}-\Delta_{\mathrm{s}}$. In the experiment, we measure the changes of the OIRD real parts $\operatorname{Re}\left\{\Delta_{\mathrm{p}}-\Delta_{\mathrm{s}}\right\}$.

In this study, we selected the polymer brush functionalized glass slide [15] as the microarry substrates, mouse immunoglobulin-G (IgG) as the target and goat anti-mouse IgG as the probe (all the IgG was purchased from KPL Inc, USA). We used conventional procedure [16] to fabricate the IgG microarrys. Firstly, the mouse IgG with different concentrations, $1,0.5$ and $0.25 \mathrm{mg} / \mathrm{mL}$, were printed at the surface of polymer brush functionalized slide using a spotting robot microsystem (Personal ArrayerTM 16, CapitalBio Corporation CA). As shown in Figure 2, every two columns is printed with $\operatorname{IgG}$ concentration decreasing from top to bottom, and each row contains five same spots. The center to center separation of the adjacent IgG spots is about 300 $\mu \mathrm{m}$, and the average diameter of the sample spots is about $100 \mu \mathrm{m}$. Secondly, the mouse IgG microarry was washed with $1 \times \mathrm{PBS}$ (phosphate buffered saline) for $5 \mathrm{~min}$ and $\mathrm{ddH}_{2} \mathrm{O}$ for $5 \mathrm{~min}$ in order to remove the redundant $\mathrm{IgG}$ and salt precipitate, and then the microarry was dried using the nitrogen. Thirdly, the IgG microarry was mounted on the reaction chamber as shown in Figure 1.

We measured the entirely interaction processes of the IgG proteins. Before interaction, we filled the reaction chamber with $1 \times \mathrm{PBS}$, and label-free measured the two dimensional scanning image of the mouse IgG microarray with different concentrations. Then, $1 \times \mathrm{PBS}$ was substituted with $0.01 \mathrm{mg} / \mathrm{mL}$ goat anti-mouse $\mathrm{IgG}$ in the reaction chamber, the detection light spot moved back and forth along the centerline of the six sample spots in left a column of the microarray, and we real-time monitored the interaction dynamic processes of the six sample spots. When the interaction finished, we again measured the two dimensional scanning image of the IgG microarray.

\section{Results and discussion}

Figure 2(a) and (b) displays the two dimensional scanning images of the OIRD $\operatorname{Re}\left\{\Delta_{\mathrm{p}}-\Delta_{\mathrm{s}}\right\}$ intensities measured before and after reaction, respectively. From Figure 2(a) and (b), one can clearly see that the gray values of sample spots in different concentrations are dropped with the concentration decreasing, indicating that it is more advanced than that of label methods, and OIRD method can label-free distinguish the different concentrations of proteins. Figure 2(c) is the differential image obtained by subtracting Figure 2(a) from Figure 2(b), showing the specificity binding between mouse IgG with different concentrations and anti-mouse IgG.

Figure 3 shows the variations of the OIRD $\operatorname{Re}\left\{\Delta_{\mathrm{p}}-\Delta_{\mathrm{s}}\right\}$ signal with time, which corresponded to the reaction dynamic processes between anti-mouse IgG and mouse IgG with different concentrations. The curves $\mathrm{A}, \mathrm{B}$, and $\mathrm{C}$ correspond to the different concentration IgGs, $1,0.5$ and 0.25 $\mathrm{mg} / \mathrm{mL}$, respectively, and the same concentration has two sample spots. The data in curve D are obtained from the slide surface without mouse IgG close to zero, meaning that anti-mouse IgG do not interact with the slide. From the curves $\mathrm{A}, \mathrm{B}$, and $\mathrm{C}$, we can see that the interaction process is an exponential behavior; the $\mathrm{IgG}$ concentration higher, the $\operatorname{Re}\left\{\Delta_{\mathrm{p}}-\Delta_{\mathrm{s}}\right\}$ signal intensity bigger. The curves A, B, and $\mathrm{C}$ tend to saturate and reach an equilibrium state

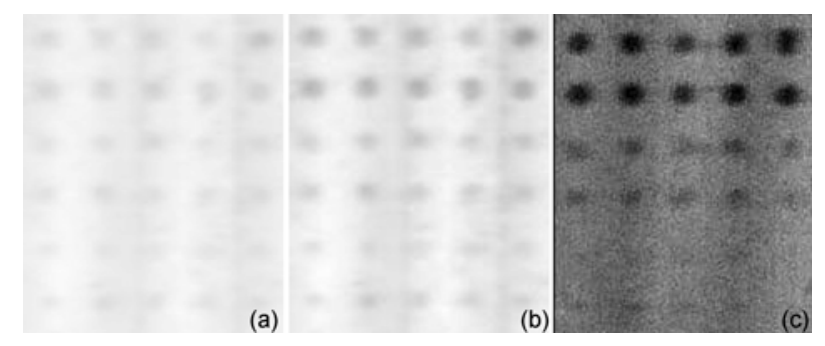

Figure 2 The two dimensional scanning images of OIRD $\operatorname{Re}\left\{\Delta_{\mathrm{p}}-\Delta_{\mathrm{s}}\right\}$ intensity signals. (a) Before reaction; (b) after reaction; (c) the differential image of OIRD $\operatorname{Re}\left\{\Delta_{\mathrm{p}}-\Delta_{\mathrm{s}}\right\}$ signal obtained by subtracting (a) from (b). 


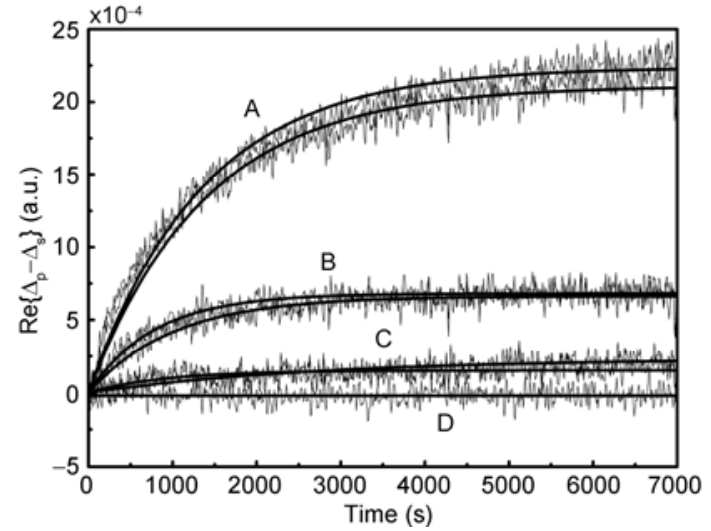

Figure 3 The variations of OIRD $\operatorname{Re}\left\{\Delta_{\mathrm{p}}-\Delta_{\mathrm{s}}\right\}$ signal with time. Curves A, $\mathrm{B}, \mathrm{C}$ and $\mathrm{D}$ correspond to different concentrations of $\operatorname{IgG}, 1,0.5,0.25$ and $0 \mathrm{mg} / \mathrm{mL}$ (from the slide surface without mouse $\mathrm{IgG}$ ), respectively.

with the time increasing, meaning the specific binding finished.

\section{Conclusion}

We measured the entire interaction process between anti-mouse IgG and mouse IgG with different concentrations, including label-free detections before and after interactions as well as label-free monitor of the interaction kinetic processes by the OIRD method, and achieved the two dimensional scanning images of the OIRD $\operatorname{Re}\left\{\Delta_{\mathrm{p}}-\Delta_{\mathrm{s}}\right\}$ intensities before and after interactions, and the curves of reaction dynamic processes. The experimental results suggest that OIRD method not only can label-free distinguish the different concentrations of proteins and detect the biological microarrays, but also can real-time monitor the kinetic processes of biomolecular interactions. Further study on the kinetic process of biomolecular interactions and the quantitative analysis of kinetic processes is planned.

This work was supported by the National Basic Research Program of
China (2007CB935701).

1 Zhu H, Bilgin M, Bangham R, et al. Global analysis of protein activities using proteome chips. Science, 2001, 293: 2101-2105

2 Espina V, Woodhouse E C, Wulfkuhle J, et al. Protein microarray detection strategies: Focus on direct detection technologies. J Immunol Methods, 2004, 290: 121-133

3 Yu X B, Xu D K, Cheng Q. Label-free detection methods for protein microarrays. Proteomics, 2006, 6: 5493-5503

4 Ray S, Mehta G, Srivastava S. Label-free detection techniques for protein microarrays: Prospects, merits and challenges. Proteomics, 2010, 10: 731-748

5 Nelson B P, Frutos A G, Brockman J M, et al. Near-infrared surface plasmon resonance measurements of ultrathin films. 1. Angle shift and SPR imaging experiments. Anal Chem, 1999, 71: 3928-3934

6 Mannelli F, Minunni A, Tombelli S, et al. Direct immobilisation of DNA probes for the development of affinity biosensors. Bioelectrochemistry, 2005, 66: 129-138

7 Fei Y Y, Landry J P, Sun Y S, et al. A novel high-throughput scanning microscope for label-free detection of protein and smallmolecule chemical microarrays. Rev Sci Instrum, 2008, 79: 013708

8 Wang X, Yuan K, Lu H, et al. Label-free detection of oligonucleotide microarrays by oblique-incidence reflectivity difference method. J Appl Phys, 2010, 107: 063109

9 Wang $\mathrm{X}, \mathrm{Lu} \mathrm{H}$, Wen J, et al. Label-free and high-throughput detection of protein microarrays by oblique-incidence reflectivity difference method. Chin Phys Lett, 2010, 27: 107801

$10 \mathrm{Lu} \mathrm{H}$, Wen J, Wang X, et al. Detection of the specific binding on protein microarrays by oblique-incidence reflectivity difference method. J Optics, 2010, 12: 095301

11 Wang X, Lu H, Dai J, et al. Real-time and label-free detection of biomolecular interactions by oblique-incidence reflectivity difference method. Chin Phys B, 2011, 20: 010704

12 Yuan K, Wang X, Lu H, et al. Label-free detection of hybridization of oligonucleotides by oblique-incidence reflectivity difference method. Sci China Phys Mech Astron, 2010, 53: 1434-1437

$13 \mathrm{Lu} \mathrm{H}$, Wen J, Wang X, et al. Detection of hybridization of protein microarrays using an oblique-incidence reflectivity difference method. Sci China Phys Mech Astron, 2010, 53: 1230-1233

14 Wen J, Lu H, Wang X, et al. Detection of protein microarrays by oblique-incidence reflectivity difference technique. Sci China Phys Mech Astron, 2010, 53: 306-309

15 Hu W, Liu Y, Lu Z, et al. Poly oligo(ethylene glycol) methacrylate-co-glycidyl methacrylate brush substrate for sensitive surface plasmon resonance imaging protein arrays. Adv Funct Mater, 2010, 20: 3497-3503

16 Liang R Q, Tan C Y, Ruan K C. Colorimetric detection of protein microarrays based on nanogold probe coupled with silver enhancement. J Immunol Methods, 2004, 285: 157-163

Open Access This article is distributed under the terms of the Creative Commons Attribution License which permits any use, distribution, and reproduction in any medium, provided the original author(s) and source are credited. 